\title{
Comparison of uncommon EGFR exon 2I L858R compound mutations with single mutation
}

\author{
This article was published in the following Dove Press journal: \\ OncoTargets and Therapy \\ 21 April 2015 \\ Number of times this article has been viewed
}

\section{Liang Peng ${ }^{1, *}$ \\ Zhigang Song 2 ,* \\ Shunchang Jiao' \\ 'Department of Oncology, Chinese PLA General Hospital, Chinese PLA Medical School, Beijing, People's Republic of China; ${ }^{2}$ Department of Pathology, Chinese PLA General Hospital, Beijing, People's Republic of China}

*These authors contributed equally to this work
Correspondence: Shunchang Jiao

Department of Oncology, Chinese PLA General Hospital, Chinese PLA Medical School, 8th Floor, ZhongLiu Building, 28\# Fuxing Road, Beijing 100853,

People's Republic of China Tel/fax +861066937878

Email shunchangschjj@163.com
Abstract: Non-small-cell lung cancer with epidermal growth factor receptor $(E G F R)$ mutation is sensitive to $E G F R$ tyrosine kinase inhibitors (TKIs). But little is known about the response to EGFR TKIs and the prognostic role of compound mutations. This study compared the uncommon EGFR exon 21 L858R compound mutations with single mutation to characterize $E G F R$ compound mutations and investigated their response to $E G F R$ TKI treatment. We retrospectively screened 799 non-small-cell lung cancer patients from August 1, 2009 to June 1, 2012 by EGFR mutation testing. EGFR mutations were detected in 443 patients, with 22 (4.97\%) compound mutations. Subsequently, six patients with EGFR exon 21 L858R compound mutations and 18 paired patients with single L858R mutation were well characterized. Finally, we also analyzed the EGFR TKI treatment response and patients' outcomes of compound or single L858R mutations. There was no differential treatment effect on the disease control rate and objective response rate between the L858R compound mutations and single mutation groups. No significant difference in overall survival or progression-free survival of these two groups was found by log-rank test. In conclusion, we demonstrated that no significant difference was detected in the response to EGFR TKIs and patients' outcomes in the compound and single mutation groups.

Keywords: non-small-cell lung cancer, EGFR mutation, compound mutations

\section{Introduction}

Lung cancer, representing the most common cancer in China, is often diagnosed at an advanced stage. Non-small-cell lung cancer (NSCLC) accounts for $80 \%-85 \%$ of lung cancers. ${ }^{1}$ Patients with advanced NSCLC achieve a modest benefit from cytotoxic chemotherapy; more specifically, the response rates of chemotherapy are $20 \%-35 \%$ and median survival is about $10-12$ months..$^{2-4}$ Recent therapeutic applications of targeted therapy have provided us with new treatment options for this disease.

Epidermal growth factor receptor $(E G F R)$ is frequently mutated in NSCLC. Mutations of the four exons, 18-21, at the EGFR tyrosine kinase domain are found, among which exon 19 and 21 mutations are the two major classic mutations. ${ }^{5-7}$ Small-molecule EGFR tyrosine kinase inhibitors (TKIs), including erlotinib and gefitinib, were the earliest drugs used in patients with advanced NSCLC in China. Recent data showed that $E G F R$ mutation was associated with an improved progression-free survival (PFS) and a high response rate to EGFR TKIs when compared with standard first-line chemotherapy in NSCLC. ${ }^{5}$

Of note, evidence has suggested that different mutation status could result in inconsistent responses to EGFR TKI treatments and also different outcomes. The Iressa Pan-Asia Study (IPASS) showed that significant differences were not found in PFS and objective response rate (ORR) with gefitinib in the exon 19 deletions and the exon 21 point mutation L858R subgroup. ${ }^{5}$ However, in the study of Won et $\mathrm{l}^{6}$ although 
no statistical differences in overall survival (OS) and ORR were observed in exon 19 deletions and L858R mutation, exon 19 deletions predicted favorable PFS following $E G F R$ TKI treatment in advanced NSCLC. Besides major EGFR mutations, there are still some rare mutations. Sequist et $\mathrm{al}^{7}$ screened 98 patients from eleven centers and concluded that exon 19 deletions and the exon 21 point mutation L858R accounted for $53 \%$ and $26 \%$ of the mutations, respectively. The remaining $21 \%$ atypical mutations included T790M in exon 20, G719A in exon 18, and L861Q in exon 21. ORR and time to progression were not statistically different among L858R and exon 19 deletion patients.

The most classic mutations have been well investigated, while few studies have focused on the clinical significance of other uncommon EGFR mutations such as compound EGFR mutations where two or even more mutations coexist in tumor. This present study aimed to improve the understanding of unknown clinical significance of the compound mutations involving the EGFR TKI L858R.

\section{Materials and methods Patient population}

This retrospective study was performed on patients with primary lung cancer, and these patients were screened by EGFR mutation testing at the Department of Pathology of The General Hospital of Chinese People's Liberation Army, Beijing, People's Republic of China from August 1, 2009 to June 1, 2012. The inclusion criteria were as follows: patients had complete medical records (including performance status, family history, smoking history, histology, treatment history, response evaluation); the necessary relevant detection data (such as histology; computed tomography [CT] scans of the head, chest, and abdomen; and blood test) were available; $E G F R$ compound mutations or single mutation at exon $21 \mathrm{~L} 858 \mathrm{R}$ were present in patients; and patients were treated with EGFR TKIs and followed up regularly. EGFR mutation testing was performed on cytologic or histologic specimens of either primary or metastatic tumors. Clinical staging was determined according to the seventh-edition American Joint Committee on Cancer (AJCC) TNM classification. The study was approved by local ethics committees at each institution and consent was obtained from the patients or their families.

\section{Treatment and evaluation}

Patients with advanced lung cancer were treated with either oral gefitinib (250 mg) or erlotinib (150 mg) per day until progression or intolerable adverse effects. Chest CT scans were performed to assess the efficacy at the beginning of treatment and then monthly. Brain CT, abdominal CT, or bone scan was performed when there were relevant symptoms in patients.

Tumor response was assessed according to the Response Evaluation Criteria in Solid Tumors (RECIST) criteria (version 1.1), which include partial response (PR), progressive disease (PD), stable disease (SD), and complete response (CR). Tumor ORR was defined as the sum of CR and PR rates. ${ }^{8}$ The disease control rate (DCR) includes $\mathrm{CR}$, $\mathrm{PR}$, and $\mathrm{SD}$ rates.

OS was measured as time from treatment to the date of death from any cause, the last follow-up, or lost to follow-up. PFS was defined as time from initiation of treatment to progression or all-cause death.

\section{Detection of EGFR mutations}

All detections were performed before treatment. Cytologic or histologic specimens from primary or metastatic tumors were used for detection. After initial pathomorphological diagnosis by pathologists using hematoxylin-eosin staining, EGFR mutations were detected by DNA direct sequencing techniques. ${ }^{9}$ In brief, paraffin blocks of NSCLC were sliced into $5 \mu \mathrm{m}$-thick sections. DNA was isolated from the sections which were baked for deparaffinization and dehydrated with gradient ethanol and then quantified and amplified by nested polymerase chain reaction (PCR) using primers of exons 18-21 of EGFR. Once purified, the PCR products were analyzed by direct DNA sequencing. Mutations were confirmed with two independent PCR reactions.

\section{Statistical analysis}

A 1:3 matched study (compound mutation cases:single mutation cases $=1: 3$ ) was performed for compound mutation and single mutation cases, and matching was performed on the characteristics of advanced stage, EGFR mutation at exon 21 L858R, use of TKIs, histology, and age.

Data analyses were performed using SPSS (v 17.0; SPSS Inc., Chicago, IL, USA). Fisher's exact test was used to compare the differences of ORR and the DCR. The survival data were analyzed by the Kaplan-Meier method, and the differences between the two groups were performed by using the log-rank test.

\section{Results}

\section{Frequency of EGFR mutations}

A total of 799 patients with lung cancer underwent EGFR mutations testing from August 1, 2009 to June 1, 2012, 
including 461 males and 338 females. Ultimately, mutations were detected in 443 patients, among which 421 (95.03\%) were single mutations and 22 (4.97\%) were compound mutations.

\section{Frequency of EGFR compound mutations harboring L858R}

Twenty-two (2.75\%) compound mutations were detected in the whole cohort, 16 of 22 patients harbored exon 21 missense mutations.

Of the 16 patients, seven cases were at stage I (T1N0M0). One of the seven patients was diagnosed as stage I (T1N0M0) right upper-lobe lung adenocarcinoma, combined with stage I (T1N0M0) right lower-lobe lung bronchoalveolar carcinoma. The patient persisted with adjuvant therapy after curative resection and used EGFR TKI treatment for 2 years; no recurrence or metastasis was found at the latest follow-up (33 months). The other six patients at stage I did not receive adjuvant therapy after curative resection with no recurrence or metastasis detected until latest follow-up. Nine patients were at advanced stage. Of the nine patients, three cases had exon 21 L833V + L833F mutations, and six cases had exon $21 \mathrm{~L} 858 \mathrm{R}$ plus another mutation. All the nine patients received EGFR TKI treatment. The main characteristics and mutation statuses are detailed in Table 1. The addition mutations, including T790M, delE746-A750, P753S, S768I, and E709K, were confirmed as somatic mutations by detection of adjacent noncancerous tissues.

\section{Comparison of baseline characteristics}

Six cases with L858R plus another mutation and 18 matched cases with single mutation were analyzed in the present study. The baseline characteristics are listed in Table 2.

No significant differences were found in age, race, sex, smoking status, performance status, histology, EGFR TKIs, and operation history between the compound mutations and single mutation groups (all $P>0.05$ ).

\section{Response and survival}

In the compound mutations group, five of the six patients achieved SD and one of the six patients achieved PR. In the single mutation group, four achieved PR, 13 achieved $\mathrm{SD}$, and one achieved PD. There was no differential treatment effect on the DCR $(6 / 6,100 \%$ versus $17 / 18,94.4 \%$; $P=1.0)$ and $\operatorname{ORR}(1 / 6,16.7 \%$ versus $4 / 18,22.2 \% ; P=0.5392)$ between the compound mutations group and single mutation group by Fisher's exact test.

The latest follow-up was performed in December 2013. The median PFS in the compound mutations group was 12.2 months. However, because the follow-up time for the single mutation was not long enough, the median PFS was not detected, while the median PFS exceeded 8 months until the latest follow-up. The PFS was not significantly different between these two groups by log-rank test $(P=0.779)$ (Figure 1).

The 1-year survival rates were $66.7 \%$ for compound mutations and $55.6 \%$ for single mutation, and the 2-year survival rates were $50 \%$ and $33.4 \%$, respectively.

The median OS for the compound mutations group was 28.6 months. Because the single mutation group did not have enough follow-up time, the median OS could not be detected, while the median OS exceeded 28 months until the latest follow-up. No statistically significant difference was found in OS between these two groups by log-rank test $(P=0.833)$ (Figure 2).

\section{Discussion}

EGFR compound mutations are uncommon mutations, the numbers of which are small. Recently, increasing studies have focused on these atypical mutations. ${ }^{10-12}$ Our study first compared the clinical significance of compound mutations with L858R plus another mutation versus single L858R mutation in Chinese lung cancer patients. The results suggested that there were no significant differences in DCR, OR, PFS, and OS between these two groups. This is the first study to focus on the response to

Table I Main characteristics of patients with exon 2 I L858R compound mutations

\begin{tabular}{|c|c|c|c|c|c|c|c|c|}
\hline Sex & $\begin{array}{l}\text { Age } \\
\text { (years) }\end{array}$ & Histology & $\begin{array}{l}\text { Smoking } \\
\text { (pack-years) }\end{array}$ & EGFR TKIs & $\begin{array}{l}\text { Objective } \\
\text { response }\end{array}$ & $\begin{array}{l}\text { PFS } \\
\text { (months) }\end{array}$ & $\begin{array}{l}\text { OS } \\
\text { (months) }\end{array}$ & Mutation \\
\hline Male & 74 & Adenocarcinoma & 0 & Gefitinib & $\mathrm{SD}$ & 8 & $8+$ & delE746-A750, L858R \\
\hline Female & 47 & Adenocarcinoma & 10 & Gefitinib & SD & 21 & 39 & P753S, L858R \\
\hline Female & 53 & Squamous carcinoma & 0 & Gefitinib & PR & 15 & $58+$ & delE746-A750, L858R \\
\hline Male & 70 & Adenocarcinoma & 20 & Gefitinib & $\mathrm{SD}$ & 6 & 6.5 & S768I, L858R \\
\hline Female & 48 & Adenocarcinoma & 0 & Gefitinib & $\mathrm{SD}$ & 10 & $18+$ & T790M, L858R \\
\hline Male & 68 & Adenocarcinoma & 80 & Gefitinib & SD & 10 & 26 & E709K, L858R \\
\hline
\end{tabular}

Abbreviations: EGFR TKIs, epidermal growth factor receptor tyrosine kinase inhibitors; PFS, progression-free survival; OS, overall survival; SD, stable disease; PR, partial response. 
Table 2 Baseline characteristics of compound mutations and single mutation

\begin{tabular}{|c|c|c|c|}
\hline Characteristics & $\begin{array}{l}\text { Compound } \\
\text { mutations }\end{array}$ & $\begin{array}{l}\text { Single } \\
\text { mutation }\end{array}$ & $P$ \\
\hline Age, mean $\pm S D$ (years) & $60 \pm 15$ & $60 \pm 15$ & $>0.05$ \\
\hline Race & Asian & Asian & $=1$ \\
\hline Sex & & & $>0.05$ \\
\hline Male & 3 & 7 & \\
\hline Female & 3 & 11 & \\
\hline Smoking status & & & $>0.05$ \\
\hline Smoking & 3 & 3 & \\
\hline None or little ${ }^{a}$ & 3 & 15 & \\
\hline ECOG PS & & & $>0.05$ \\
\hline 0 & 5 & 15 & \\
\hline 1 & I & 2 & \\
\hline 2 & 0 & 1 & \\
\hline Histology & & & $>0.05$ \\
\hline Adenocarcinoma & 5 & 16 & \\
\hline Squamous carcinoma & I & 2 & \\
\hline EGFR TKIs & & & $>0.05$ \\
\hline Gefitinib & 6 & 12 & \\
\hline Erlotinib & 0 & 4 & \\
\hline Other & 0 & 2 & \\
\hline Operation history & & & $>0.05$ \\
\hline None & 2 & 9 & \\
\hline Yes & 4 & 9 & \\
\hline Line $^{b}$ & & & $>0.05$ \\
\hline First & 3 & 13 & \\
\hline Second & 3 & 5 & \\
\hline
\end{tabular}

Notes: a No smoking or smoking less than 10 pack-years. ${ }^{b}$ First: TKI as first-line therapy; second: TKI as second-line therapy.

Abbreviations: ECOG PS, Eastern Cooperative Oncology Group performance status; EGFR TKIs, epidermal growth factor receptor tyrosine kinase inhibitors.

EGFR TKIs of compound L858R mutations and compare the difference between compound mutations and single mutation in Chinese patients, as far as we know. This may be helpful in the development of strategies for optimizing the use of EGFR TKIs.

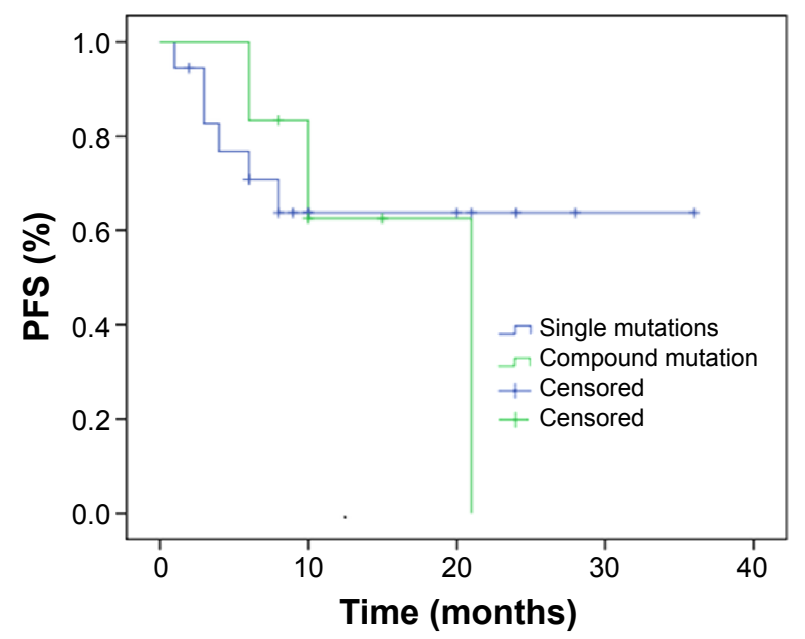

Figure I Survival curves of progression-free survival (PFS) according to the mutation status of EGFR exon 21 L858R.



Figure 2 Survival curves of overall survival (OS) according to the mutation status of EGFR exon 2I L858R.

EGFR mutations were found in $10 \%-20 \%$ and $30 \%-60 \%$ of Caucasian and Asian NSCLC patients, respectively, and EGFR mutations were more frequently detected in women, nonsmokers, and patients with adenocarcinoma histology. ${ }^{13-17}$ The most frequent mutations are involved in exon 19 and exon 21 (L858R), and these mutations have been demonstrated to be associated with better response rates and PFS with use of EGFR TKIs versus standard chemotherapies. ${ }^{18,19}$ However, compared with common $E G F R$ mutations, relatively little is known about rare EGFR compound mutations. In our study, $22(2.75 \%)$ patients with compound mutations were detected. Similarly, Wu et al ${ }^{10}$ also reported that 20 (3.19\%) cases had compound mutations. In addition, Kobayashi et a ${ }^{11}$ detected the EGFR mutation pattern in 79 NSCLC cases, and then found that eleven (14\%) patients had compound mutations. In Liu et al's study, multiple mutations were detected in 21 patients $(21 / 140,15.0 \%)$ from 140 mutation cases. ${ }^{12}$ According to the above studies, the compound mutations had a low incidence rate in NSCLC cases.

The response to EGFR TKIs and the effects on patients' clinical outcomes, of compound mutations, have not been completely defined. Kobayashi et $\mathrm{a}^{11}$ reported three patients with L858R compound mutations, among which cases with L747V plus L858R and R776H plus L858R had PR and the case with L858R plus A871G had PD to EGFR TKIs, with $6+, 3+$, and 2 months for PFS and 6+, 3+, and 3 months for OS, respectively. In the present study, six advanced NSCLC patients with compound L858R mutations received TKI treatments; five of the six patients had SD and one patient had PR. The median PFS and OS for these six patients were 12.2 months and 28.6 months, respectively.

Research has demonstrated that T790M is closely related to EGFR TKI resistance ${ }^{20,21}$ and considered as a second 
somatic mutation located at exon $20 .{ }^{22} \mathrm{Yu}$ et al found that patients with baseline EGFR T790M mutation had limited benefit from $E G F R$ TKI treatment. ${ }^{23}$ In addition, Kim et $\mathrm{al}^{24}$ reported that one patient with L858R + T790M mutations was administrated EGFR TKI and had PD within 1 month, with PFS 1 month and OS 17.4 months. However, in our study, one patient with T790M + L858R mutations had SD, with PFS of 10 months and OS of 18 months (still alive). The different outcomes of these studies indicate that there were other factors involved with and affecting patients' responses to treatment and outcomes.

Furthermore, we also found four other compound mutations, including L858R + delE746-A750/P753S/S768I/ E709K, and these compound mutations were not reported in other studies. Previous study has shown that delE746A750 was a common activating EGFR somatic mutations in exon 19 and played a key role in drug sensitivity to gefitinib. ${ }^{25,26} \mathrm{~S} 768 \mathrm{I}$ was a rare $E G F R$ somatic mutation in exon 20 and showed a good clinical response to gefitinib. ${ }^{27,28}$ Consistently, we found that patients with L858R + delE746A750/S768I compound mutations had a good objective response to EGFR TKIs. P753S and E709K, rare somatic EGFR mutations in exon 19 and 18, respectively, were found in patients with cutaneous squamous cell carcinoma and head and neck squamous cell carcinoma, respectively. ${ }^{29,30}$ However, our study demonstrates that L858R + P753S/ E709K compound mutations had a good objective response to EGFR TKIs. These results indicate that L858R might play a primary role in objective response to EGFR TKIs, and the mechanism of interaction between compound mutations should be further investigated.

Our study had some limitations. Because of the rare incidence of compound mutations, only 22 cases were involved in the study. This might increase the opportunity of false negative error. As a retrospective study, the treatment strategies of patients were not consistent. Some patients received $E G F R$ TKIs as first-line therapy, while this was second- or third-line therapy in others. This inconsistency may influence the response to treatment and patients' outcomes. Further studies including more patients with compound mutations are needed.

\section{Conclusion}

We compared the clinical significance and the response to treatment of EGFR TKIs of the rare L858R compound mutations and L858R single mutation. We demonstrated that no significant differences were detected in the response to EGFR TKIs and patients' outcomes between the compound and single mutation groups.

\section{Acknowledgment}

None.

\section{Author contributions}

Liang Peng and Zhigang Song: acquisition of data, design of the study, carrying out the study, and critical revision. Shunchang Jiao: manuscript writing and acquisition of data.

\section{Disclosure}

The authors report no conflicts of interest in this work.

\section{References}

1. Peters S, Adjei AA, Gridelli C, Reck M, Kerr K, Felip E; ESMO Guidelines Working Group. Metastatic non-small-cell lung cancer (NSCLC): ESMO Clinical Practice Guidelines for diagnosis, treatment and follow-up. Ann Oncol. 2012;23 Suppl 7:vii56-vii64.

2. Sandler A, Gray R, Perry MC, et al. Paclitaxel-carboplatin alone or with bevacizumab for non-small-cell lung cancer. N Engl J Med. 2006; 355(24):2542-2550.

3. Schiller JH, Harrington D, Belani CP, et al; Eastern Cooperative Oncology Group. Comparison of four chemotherapy regimens for advanced nonsmall-cell lung cancer. N Engl J Med. 2002;346(2):92-98.

4. Fossella F, Pereira JR, von Pawel J, et al. Randomized, multinational, phase III study of docetaxel plus platinum combinations versus vinorelbine plus cisplatin for advanced non-small-cell lung cancer: the TAX 326 study group. J Clin Oncol. 2003;21(16):3016-3024.

5. Fukuoka M, Wu YL, Thongprasert S, et al. Biomarker analyses and final overall survival results from a phase III, randomized, open-label, first-line study of gefitinib versus carboplatin/paclitaxel in clinically selected patients with advanced non-small-cell lung cancer in Asia (IPASS). J Clin Oncol. 2011;29(21):2866-2874.

6. Won YW, Han JY, Lee GK, et al. Comparison of clinical outcome of patients with non-small-cell lung cancer harbouring epidermal growth factor receptor exon 19 or exon 21 mutations. J Clin Pathol. 2011;64(11):947-952.

7. Sequist LV, Martins RG, Spigel D, et al. First-line gefitinib in patients with advanced non-small-cell lung cancer harboring somatic EGFR mutations. J Clin Oncol. 2008;26(15):2442-2449.

8. Edeline J, Boucher E, Rolland Y, et al. Comparison of tumor response by Response Evaluation Criteria in Solid Tumors (RECIST) and modified RECIST in patients treated with sorafenib for hepatocellular carcinoma. Cancer. 2012;118(1):147-156.

9. Metzger B, Chambeau L, Begon DY, et al. The human epidermal growth factor receptor (EGFR) gene in European patients with advanced colorectal cancer harbors infrequent mutations in its tyrosine kinase domain. BMC Med Genet. 2011;12:144.

10. Wu JY, Yu CJ, Chang YC, Yang CH, Shih JY, Yang PC. Effectiveness of tyrosine kinase inhibitors on "uncommon" epidermal growth factor receptor mutations of unknown clinical significance in non-small cell lung cancer. Clin Cancer Res. 2011;17(11):3812-3821.

11. Kobayashi S, Canepa HM, Bailey AS, et al. Compound EGFR mutations and response to EGFR tyrosine kinase inhibitors. J Thorac Oncol. 2013;8(1):45-51.

12. Liu Y, Wu BQ, Zhong HH, Hui P, Fang WG. Screening for EGFR and KRAS mutations in non-small cell lung carcinomas using DNA extraction by hydrothermal pressure coupled with PCR-based direct sequencing. Int J Clin Exp Pathol. 2013;6(9):1880-1889.

13. Mitsudomi T, Kosaka T, Endoh H, et al. Mutations of the epidermal growth factor receptor gene predict prolonged survival after gefitinib treatment in patients with non-small-cell lung cancer with postoperative recurrence. J Clin Oncol. 2005;23(11):2513-2520.

14. Mok TS, Wu YL, Thongprasert S, et al. Gefitinib or carboplatinpaclitaxel in pulmonary adenocarcinoma. $N$ Engl J Med. 2009; 361(10):947-957. 
15. Rosell R, Moran T, Queralt C, et al; Spanish Lung Cancer Group. Screening for epidermal growth factor receptor mutations in lung cancer. N Engl J Med. 2009;361(10):958-967.

16. Shigematsu H, Lin L, Takahashi T, et al. Clinical and biological features associated with epidermal growth factor receptor gene mutations in lung cancers. J Natl Cancer Inst. 2005;97(5):339-346.

17. Shih JY, Gow CH, Yu CJ, et al. Epidermal growth factor receptor mutations in needle biopsy/aspiration samples predict response to gefitinib therapy and survival of patients with advanced nonsmall cell lung cancer. Int J Cancer. 2006;118(4):963-969.

18. Gaughan EM, Costa DB. Genotype-driven therapies for non-small cell lung cancer: focus on EGFR, KRAS and ALK gene abnormalities. Ther Adv Med Oncol. 2011;3(3):113-125.

19. Rosell R, Carcereny E, Gervais R, et al; Spanish Lung Cancer Group in collaboration with Groupe Français de Pneumo-Cancérologie and Associazione Italiana Oncologia Toracica. Erlotinib versus standard chemotherapy as first-line treatment for European patients with advanced EGFR mutation-positive non-small-cell lung cancer (EURTAC): a multicentre, open-label, randomised phase 3 trial. Lancet Oncol. 2012;13(3):239-246.

20. Arcila ME, Oxnard GR, Nafa K, et al. Rebiopsy of lung cancer patients with acquired resistance to EGFR inhibitors and enhanced detection of the T790M mutation using a locked nucleic acid-based assay. Clin Cancer Res. 2011;17(5):1169-1180.

21. Chmielecki J, Foo J, Oxnard GR, et al. Optimization of dosing for EGFR-mutant non-small cell lung cancer with evolutionary cancer modeling. Sci Transl Med. 2011;3(90):90ra59.

22. Pao W, Miller VA, Politi KA, et al. Acquired resistance of lung adenocarcinomas to gefitinib or erlotinib is associated with a second mutation in the EGFR kinase domain. PLoS Med. 2005;2(3):e73.
23. Yu H, Arcila M, Hellmann M, Kris M, Ladanyi M, Riely G. Poor response to erlotinib in patients with tumors containing baseline EGFR T790M mutations found by routine clinical molecular testing. Ann Oncol. 2014;25(2):423-428.

24. Kim HR, Cho BC, Shim HS, et al. Prediction for response duration to epidermal growth factor receptor-tyrosine kinase inhibitors in EGFR mutated never smoker lung adenocarcinoma. Lung Cancer. 2014;83(3):374-382.

25. Lynch TJ, Bell DW, Sordella R, et al. Activating mutations in the epidermal growth factor receptor underlying responsiveness of non-small-cell lung cancer to gefitinib. $N$ Engl J Med. 2004;350(21):2129-2139.

26. Paez JG, Jänne PA, Lee JC, et al. EGFR mutations in lung cancer: correlation with clinical response to gefitinib therapy. Science. 2004; 304(5676):1497-1500.

27. Boespflug A, Couraud S, Bringuier PP, et al. Primary lung adenocarcinoma occurring in a PTEN related syndrome (Cowden's disease): routine EGFR sequencing also highlights two rare somatic mutations S768I and V769L. Lung Cancer. 2013;79(3):318-320.

28. Masago K, Fujita S, Irisa K, et al. Good clinical response to gefitinib in a non-small cell lung cancer patient harboring a rare somatic epidermal growth factor gene point mutation; codon 768 AGC $>$ ATC in exon 20 (S768I). Jpn J Clin Oncol. 2010;40(11):1105-1109.

29. Ganesan P, Ali SM, Wang K, et al. Epidermal growth factor receptor P753S mutation in cutaneous squamous cell carcinoma responsive to cetuximab-based therapy. J Clin Oncol. Epub 2014 Jun 16.

30. Hama T, Yuza Y, Saito Y, et al. Prognostic significance of epidermal growth factor receptor phosphorylation and mutation in head and neck squamous cell carcinoma. Oncologist. 2009;14(9):900-908.
OncoTargets and Therapy

\section{Publish your work in this journal}

OncoTargets and Therapy is an international, peer-reviewed, open access journal focusing on the pathological basis of all cancers, potential targets for therapy and treatment protocols employed to improve the management of cancer patients. The journal also focuses on the impact of management programs and new therapeutic agents and protocols on

\section{Dovepress}

patient perspectives such as quality of life, adherence and satisfaction The manuscript management system is completely online and includes a very quick and fair peer-review system, which is all easy to use. Visit http://www.dovepress.com/testimonials.php to read real quotes from published authors. 